\title{
LA SPECTACULARISATION DES ÉMISSIONS POLITIQUES DANS LES TÉLÉVISIONS EUROPÉENNES
}

\begin{abstract}
Marc Lits ${ }^{1}$
Depuis plusieurs années, le discours politique connaît des évolutions radicales, liées à la transformation de l'espace public, à l'évolution des rapports entre citoyens et mandataires publics, et au rôle grandissant des médias, particulièrement audiovisuels, dans la communication politique. Diverses études ont déjà été publiées sur cette question, dans le monde anglo-américain et francophone, et il semble opportun de s'interroger aujourd'hui sur les rapports nouveaux qui se construisent dans l'interaction entre politique et médias en Communauté française. En effet, depuis quelques années, la couverture médiatique des événements et des personnalités politiques semble évoluer, tant au nord qu'au sud du pays.

Pour en prendre quelques exemples, les émissions strictement politiques semblent de moins en moins présentes à la télévision, et les personnalités politiques apparaissent plus volontiers dans d'autres environnements, jugés plus attractifs pour le téléspectateur. Pour se cantonner à des exemples français, les émissions politiques du dimanche soir qui ont longtemps été présentes à TF1 ont disparu, pendant que les hommes politiques préféraient apparaitre dans le divertissement du dimanche

1 Marc Lits est professeur à l'École de Journalisme de Louvain (EJL) et directeur de l'Observatoire du récit médiatique (ORM) au Département de communication de l'UCL.
\end{abstract}

Recherches en communication, $\mathrm{n}^{\circ} 24$ (2005). 
après-midi animé par Michel Drucker sur France 2. Mais au moment d'écrire cela, il faut constater qu'une chaîne privée belge replace en début de soirée une grande émission politique, le vendredi soir, avec un succès d'audience incontestable.

En outre, la presse people a ouvert ses colonnes aux personnalités politiques, dans le nord du pays, mais aussi, dans une moindre mesure, du côté francophone. Un mariage, une maternité, des révélations sur une vie de couple heureuse ou connaissant des remous : tous ces sujets se retrouvent en pages intérieures ou en couverture des magazines, voire de certains quotidiens. Il n'est jusqu'aux émissions électorales, traditionnellement très conformistes dans leur traitement télévisuel, qui ont voulu modifier leur approche des thématiques politiques en les personnalisant, en les mettant en scène.

\section{Quelques limites}

C'est dans ce contexte qu'il s'agit d'examiner la manière dont évoluent le contenu et le traitement formel des émissions.

Le premier problème réside dans la définition même de l'objet d'étude. Qu'entend-on par spectacularisation des émissions politiques ? Y a-t-il des critères objectivables qui permettent de définir cette notion, en se fondant sur l'analyse des discours médiatiques proposés, ou sur leur traitement télévisuel ? Les risques de positionnement subjectif sont très grands, et l'analyse pourrait très rapidement être supplantée par une mise en avant de jugements extrêmement subjectifs, davantage liés aux options politiques et médiatiques de l'analyste qu'à une approche systématisée, fondée sur des paramètres d'observation indiscutables. C'est pourquoi nous ne définirons pas a priori les notions de spectacularisation, de mise en scène, mais nous tenterons de décrire au plus près les transformations contemporaines du discours médiatique, en croisant ces analyses avec les paroles d'acteurs, tant politiques que journalistiques. Et c'est a posteriori que nous pourrons identifier les changements, en chercher les raisons, et proposer un état des lieux à partir duquel chacun pourra en tirer les interprétations qu'il souhaite, fondées sur des données objectivées.

L'autre limite de cette étude réside dans la double restriction qui est à sa base, par rapport à une vision politique globale de nos évolutions sociétales, et par rapport à l'ensemble du système médiatique. Il 
ne serait pas correct, en termes d'analyse des évolutions de nos sociétés, de considérer que les programmes politiques télévisuels peuvent s'analyser de manière autonome, et constituer en soi un objet révélateur des transformations sociales. Ce que dit la télévision, ce que disent les politiques à la télévision, ces deux objets déjà assez différents ne peuvent être analysés indépendamment des cadres sociaux dans lesquels ils s'inscrivent. C'est pourquoi plusieurs ancrages contextuels sont prévus en début et en fin d'analyse. Il s'agit de saisir, au moins brièvement, en quoi la gestion des affaires publiques et la communication des choix politiques se transforment fondamentalement dans une société qui semble se défier de la politique, où les individualismes vont croissant, où la télévision modifie aussi la manière dont les politiques parlent à leurs électeurs ou à l'ensemble des citoyens. Il faut aussi, de manière parallèle, montrer que ces transformations ne sont pas si récentes, qu'elles s'inscrivent dans une histoire de l'écriture télévisuelle. Depuis plus de cinquante ans, télévision et politique entretiennent des rapports complexes qu'il est nécessaire de resituer. Par ailleurs, la mise en forme télévisuelle a elle aussi évolué, sous la pression des transformations sociales (le rapport au privé n'est plus le même qu'il y a vingt ans) et des évolutions technologiques (l'arrivée de la couleur, les possibilités du direct, la caméra légère transforment le traitement et la scénarisation des émissions).

Si la politique a changé, si la télévision d'aujourd'hui n'est plus celle d'il y a dix ans, l'ensemble des médias a aussi connu des transformations notables. La presse quotidienne d'opinion a quasiment disparu en Belgique, les magazines de télévision font les meilleures ventes, les sujets dits "people" sont de plus en plus fréquents, dans la presse de proximité comme dans les quotidiens dits "de référence". C'est une évolution qu'il faut prendre en compte, d'autant plus que c'est probablement dans ces lieux-là que la spectacularisation du politique est la plus flagrante. C'est dans son quotidien ou son hebdomadaire que le grand public découvre les amours plus ou moins cachées des ministres et des parlementaires. Si l'on veut donc s'interroger sur la manière dont les médias personnalisent actuellement les sujets politiques, privilégient parfois l'anecdote au détriment de l'analyse, la vie privée plutôt que le contenu de choix de société posés par des partis différents, il faudrait prendre en compte les transformations de l'ensemble du système médiatique. Ce serait d'autant plus nécessaire que les médias fonctionnent de plus en plus dans une logique de boucle, l'un reprenant les informations 
de l'autre, dans une chaîne sans fin qui accroît l'effet de médiatisation. Mais la présente étude se bornera à la télévision.

L'analyse de la présence du personnel politique dans les programmes de télévision, que ce soit dans les émissions générales d'information, dans les émissions politiques ou dans des programmes autres, ne peut être comprise qu'en étant inscrite dans une double histoire, celle du média télévisuel et celle de la communication politique.

L'histoire de la télévision, saisie dans ses transformations successives depuis plus de cinquante ans, fait ressortir deux phénomènes essentiels pour éclairer cette recherche : les rapports entre politique et télévision, l'évolution technique des programmes et de leurs contenus. Dès leur création, les offices de radio-télévision ont entretenu des relations complexes et difficiles avec le pouvoir politique. Ils en dépendent, ils doivent laisser s'exprimer les autorités comme et quand elles le souhaitent, ou leur ouvrir complaisamment leur micro, mais ils cherchent toujours à s'autonomiser, à disposer d'une réelle autonomie rédactionnelle. Dès lors, si la télévision apparaissait au départ comme une simple tribune offerte aux ministres, au chef du gouvernement ou de l'Etat, peu à peu, les journalistes se sont posés tantôt en interviewers, tantôt en contradicteurs, en porte-parole de l'opinion publique, en animateurs de débats.

Parallèlement à cette progressive conquête de l'indépendance du média face au pouvoir politique, à mettre aussi en lien avec l'émergence des émetteurs privés, acceptés voire encouragés par les pouvoirs publics, les réalisations télévisées ont aussi évolué sans cesse, parce que la technique le permettait, et que l'écriture télévisuelle se transformait, pour s'adapter à de nouveaux publics, à des concurrences internes (public vs privé) ou externes (le cinéma, Internet, la presse magazine...). La couleur remplace le noir et blanc, le débat contradictoire rend obsolète le discours long cadré en plan moyen face caméra, l'information est contaminée par des procédés de tournage inspirés des émissions de divertissement. La préoccupation devient aussi moins centrée sur l'offre (la télévision se fixe des objectifs relatifs à la formation et à l'information et réalise des programmes pour répondre à ces objectifs) que sur la demande (quel programme touchera le plus grand nombre et permettra de réaliser la meilleure audience et la part de marché la plus importante ?), dans un univers de plus en plus concurrentiel.

Dans le même temps, la place de la politique dans la société a évolué, le rapport qui se tisse entre les hommes politiques et les citoyens (qui sont à la fois des électeurs et des consommateurs médiatiques) 
est différent d'il y a vingt ou trente ans, et les hommes politiques ont professionnalisé leur communication publique. Dans l'interview que le ministre de l'Intérieur français Nicolas Sarkozy accordait à Denis Jeanbar dans L'Express du 17 novembre 2005, il expliquait très clairement les raisons pour lesquelles il avait utilisé quelques termes forts pour désigner les événements qui eurent lieu à ce moment dans les banlieues françaises :

La sémantique, ça compte. Les idées, les mots, les concepts servent à déverrouiller l'action. Je prends une image: la communication est à l'action ce que l'aviation est à l'infanterie ; l'aviation doit passer pour que l'infanterie puisse sortir ; c'est lorsqu'on a gagné la bataille de la communication qu'on peut commencer à agir. Il y a vingt-cinq ans, on agissait puis on communiquait; à présent, tout a changé, c'est parce qu'on a communiqué et qu'on s'est fait comprendre qu'on peut agir.

Si le propos est radical dans la priorité qu'il met à la communication, il illustre bien comment, désormais, tout projet politique doit intégrer, dès le départ, une stratégie de communication dans laquelle la présence médiatique, particulièrement en télévision, sera déterminante. Dès lors, l'homme politique acceptera souvent les propositions qui lui sont faites par des journalistes ou des animateurs de programmes, sachant de quelles retombées il pourra bénéficier si son image et son discours sont ainsi proposés à plusieurs centaines de milliers de téléspectateurs.

Les éditeurs de services et les hommes politiques interrogés pour l'enquête qui a donné lieu à ce dossier convergent sur ce point : rares sont les responsables politiques qui refusent une invitation sur un plateau de télévision, quel que soit le type de programmes. Il y a donc là des jeux de séduction ou de pouvoir réciproques qui se mettent en place et qui rendent les deux parties solidaires de ce qui est proposé dans les programmes, que ceux-ci soient strictement politiques ou d'un tout autre ordre.

Sans empiéter sur ce que proposent les analyses qui sont présentes dans ce dossier, il est cependant possible d'en dégager les principaux enjeux, les tendances lourdes actuelles, telles qu'elles transparaissent surtout dans l'analyse des programmes belges. 


\section{Qu'est-ce que le spectaculaire ?}

Ces évolutions conjointes de l'écriture télévisuelle et de la communication politique ont donc des implications fortes sur les programmes dans lesquels se trouvent des hommes politiques, ce qui peut amener certains à parler de spectacularisation du politique. Mais ce terme est assez ambigu, à la fois parce qu'il est difficile à définir strictement et parce qu'il est porteur de connotations négatives renvoyant au concept d'infotainment.

\section{Un effet de modernisation}

Les transformations de l'écriture télévisuelle portent aussi sur les programmes politiques, et la télévision ne se cantonne plus, aujourd'hui, à faire de la radio en images. Elle construit donc ses émissions en travaillant le rythme, les éclairages, les cadrages, les décors, en multipliant l'alternance des séquences, le mode d'interpellation des invités, le recours au direct, à du matériel léger qui permet une plus grande souplesse d'enregistrement. Tous ces éléments sont réfléchis et préparés par le journaliste et le réalisateur qui vont proposer un programme avec une dynamique interne. Ce qui est donc parfois qualifié de spectacularisation relève pour une part non négligeable d'une modernisation des logiques de réalisation, choisie pour lutter contre un risque de monotonie lié à l'image des émissions politiques traditionnelles.

Certaines émissions "concédées" aux partis politiques ou aux mouvements associatifs restent encore réalisées dans une facture très classique, avec un présentateur statique et des invités se succédant pour de longs monologues. Mais c'est en général dû à un manque de moyens plutôt qu'à une volonté de garder le rythme traditionnel de la tribune politique. Certains partis politiques ont d'ailleurs renoncé à utiliser l'espace qui leur est réservé, jugeant ce mode de communication dépassé, tandis que d'autres ont essayé de le renouveler, en recourant à des équipes de réalisation professionnelles. Les temps de parole octroyés aux partis démocratiques lors des campagnes électorales sont d'ailleurs révélateurs de cette double logique: certains restent très statiques, présentant des candidats parlant face à la caméra devant un fond neutre, alors que d'autres prévoient une mise en scène de ces séquences. Enfin, il faut préciser que la modernisation demande des moyens techniques et financiers importants, dont ne disposent pas tous les éditeurs. Dans leur quasi-totalité, les télévisions locales et communautaires sont à l'abri de 
la tentation de la spectacularisation, surtout parce qu'elles n'ont pas la possibilité budgétaire d'investir dans ce type de réalisation.

\section{Un changement de contenu}

Le premier niveau de la spectacularisation se trouve d'abord dans des choix de mise en forme, voire de mise en scène de l'information politique et de ses acteurs. Mais il est évident que l'évolution de la mise en forme a un impact sur les contenus qui seront intégrés dans ce nouveau type de productions. Le ton, le rythme, l'enchaînement des séquences vont déterminer une certaine manière d'impliquer les intervenants politiques, et amener aussi à privilégier un certain type de sujets.

La manière dont les journalistes vont interviewer les politiques présents en studio a évolué dans la forme (questions plus directes, temps limité de réponse, interruption par le journaliste de tout discours long, mise en concurrence de plusieurs intervenants qui se coupent la parole, encouragement de la dimension polémique plus spectaculaire que la succession des points de vue...) et dans les thèmes proposés. Certaines émissions politiques, les débats du dimanche midi en particulier, vont être centrées davantage sur des sujets de société que sur des questions politiques au sens strict, même si la frontière entre ces deux catégories est très poreuse. Le port du voile, le respect des différences religieuses ou culturelles, la légalisation des drogues douces, sont autant de thématiques qui peuvent être abordées par des représentants de la société civile, souvent invités sur ces plateaux, mais qui sont aussi liées à des propositions ou des projets de loi. Dans ce cas, c'est le journaliste, par ses questions, par la tonalité qu'il donne au débat, qui l'orientera plutôt dans une direction ou dans une autre. Cette évolution est d'ailleurs reconnue par les éditeurs de services, qui la revendiquent explicitement comme une stratégie destinée à retrouver des téléspectateurs de moins en moins intéressés à la chose publique. Là où les débats institutionnels les font fuir, des questions relatives au "vivre ensemble" intéressent encore le public.

D'un autre côté, cette volonté d'interpeller plus directement les intervenants politiques est présentée par les journalistes comme une manière d'éviter la langue de bois de leurs invités. En les bousculant, en les déstabilisant, par exemple quand ils sont amenés dans un lieu qu'ils ne connaissent pas à l'avance, ils seront obligés de réagir spontanément, hors de toute stratégie communicationnelle préparée. 


\section{Le rapport vie publique/vie privée}

Cette approche plus personnalisée peut entraîner un déplacement de l'angle d'attaque de l'échange entre les journalistes et les politiques, dans la mesure où les questions relatives à des programmes, à des options politiques pourront être relayées à l'arrière-plan par rapport à l'expression d'une sensibilité personnelle. Dans ce cas, ce n'est plus le personnel politique qui est invité à réagir au titre de la fonction qu'il occupe, c'est la personne elle-même qui va donner son sentiment, en tant qu'individu sensibilisé à titre strictement personnel par le thème abordé.

Il faut d'abord rappeler que cet estompement de la frontière entre personne privée et personnalité publique n'est pas spécifique du discours télévisuel. Il s'est déjà manifesté dans l'affichage électoral, quand les candidats ont estimé (sur la recommandation de leurs conseillers en communication) que se montrer avec leur famille donnerait d'eux une image plus accessible, plus sympathique ; il a ensuite gagné les magazines (de Paris Match à La Libre Match, mais aussi dans les magazines de télévision comme TéléMoustique ou Ciné Télé Revue, ou les magazines féminins tels que Flair ou Femmes d'aujourd'hui), puis certains titres de la presse quotidienne. La télévision s'est donc inscrite dans une évolution plus large liée à la transformation de l'espace public où les distinctions entre sphère publique, privée et intime se recomposent autrement.

Cette question est centrale, comme la suivante, par rapport aux enjeux éthiques et sociétaux de la spectacularisation du débat politique. L'argument des tenants de l'ouverture maximale à la dimension privée tient en deux points: les hommes politiques sont des personnages publics ; montrer leurs aspects plus privés les humanise auprès du téléspectateur. Le premier point montre combien la fonction politique s'est transformée, amenant l'homme politique à sortir de son rôle strict de mandataire public élu par un ensemble de citoyens pour exercer une responsabilité à un des niveaux du pouvoir exécutif ou législatif, pour devenir une personnalité publique, exposée au regard de ses concitoyens, mais aussi des médias. Il serait donc tenu, puisque personnage public, de livrer aux médias, ou de leur laisser saisir, des aspects qui ne sont plus liés à sa fonction mais à sa personne ou à celle de ses proches.

L'autre point renvoie davantage à des stratégies de communication qui mettent moins en cause les médias (quand ils avancent l'argument 
précédent) que les politiques eux-mêmes. Il y aurait une espèce d'intérêt partagé par les deux parties : en montrant l'homme politique sous des aspects plus proches, la télévision toucherait des publics rétifs à la chose politique mais sensibles à la dimension "people" des personnalités de la vie publique (une sorte d'effet Place royale, en quelque sorte); en se montrant sous un jour favorable, l'homme politique touche une frange de l'électorat qui ne s'intéresse pas au programme qu'il a à proposer mais qui se prononcera selon un critère de sympathie personnelle. Cela explique pourquoi les hommes politiques n'ont guère de réticence à passer dans des émissions où ils savent qu'ils devront dévoiler une part de leur intimité.

\section{Le mélange des genres}

Cette forme de personnalisation est en lien direct avec l'autre trait central constitutif de la mise en spectacle des émissions politiques. En s'exposant ainsi aux caméras, le politique va se retrouver dans deux types de programmes assez différents de ce qui lui était traditionnellement proposé : des émissions politiques contaminées par le jeu et la fiction, et des émissions non politiques. Quand RTL-TVI propose Face à l'électeur ou Pour qui voter ?, et que la RTBF montre C'est ma voix ou Destination élections, il s'agit clairement de programmes politiques, proposés d'ailleurs dans la période très réglementée de la campagne électorale. Il n'y a donc pas de doute à avoir pour cataloguer ces émissions. Mais leur forme les tire tantôt vers l'univers du divertissement (un quiz télévisé, une forme de télé-réalité, des demandes de classement...), tantôt celui de la fiction (musiques de séries TV, montage recourant au split screen...). Cependant, dans tous les cas étudiés, si la forme télévisuelle sort du modèle traditionnel, celle-ci n'apparaît jamais, ni dans l'analyse réalisée a posteriori, ni dans les déclarations des éditeurs de services, ni dans les réactions des politiciens interrogés, comme une fin en soi. L'habillage ludique est destiné à rendre plus accessible le contenu, à toucher un public de moins en moins intéressé par les questions politiques, et qui est habitué à un certain type de format télévisuel quand il consomme les autres programmes quotidiens. Il s'agit donc d'inscrire les émissions politiques dans une écriture qui a gagné l'ensemble de la télévision, par souci de contact avec le plus grand nombre des téléspectateurs.

L'autre face du mélange des genres est plus délicate, puisqu'elle est relative à l'apparition d'hommes politiques dans des émissions qui 
ne relèvent plus de ce registre-là. Cela va du talk-show où les invités appartiennent à différents secteurs de la société (chanteurs et vedettes du show-business, écrivains et intellectuels, porte-parole d'associations et mouvements divers, voire individus isolés intervenant comme témoins) aux émissions de jeux et de variétés. Les raisons de leur présence dans cet environnement qui dépasse le cadre de leur fonction politique sont diverses, mais elles répondent toutes à un seul principe : l'homme politique est désormais un homme public, et à ce titre il est sollicité comme d'autres personnalités pour figurer dans des émissions organisées autour de la mise en spectacle de personnes connues par le plus grand nombre de téléspectateurs. Nul n'échappe à cette évolution, du cardinal Danneels passant aux Allumés.be à Justine Henin recevant une "femme de cristal".

Le phénomène est très répandu en France où le personnel politique est fréquemment invité dans les talk-shows et émissions de variété, répondant tantôt à des interviews "hard" chez Ardisson, tantôt se risquant à des séquences humoristiques, comme Jack Lang dansant sur le sketch de la drague du couple Bedos-Daumier. En Communauté française, ce type de programmes est plus rare. Il n'existe pas d'équivalent aux talk-shows de Thierry Ardisson ou Marc-Olivier Fogel, aux divertissements animés par un Laurent Ruquier ou un Arthur, voire d'émission plus familiale mêlant invités du monde du spectacle et de la politique comme le fait Michel Drucker le dimanche après-midi. Dès lors, les risques d'exposition spectaculaire des hommes politiques en sont très limités. Au point que la moindre apparition d'une personnalité politique dans une émission de jeu comme Génies en herbe ou dans une émission de collecte de dons comme Cap 48 entraîne des commentaires dans la presse quotidienne, dans les jours qui suivent. C'est un signe du caractère exceptionnel de ces manifestations, alors que celles-ci sont devenues banales dans les autres pays, où elles ne suscitent plus guère de réactions.

Il n'empêche que si ces mélanges de genres sont rares et relativement contrôlés par les éditeurs de services, cela ne dispense pas de mener une réflexion sur les enjeux déontologiques, éthiques et politiques de pareille évolution. La lecture positive qu'on peut en faire repose sur le postulat d'un nécessaire rapprochement entre les responsables politiques et les citoyens, entre lesquels un fossé semble s'être creusé. Dès lors, tout ce qui peut montrer que l'homme politique n'est pas un être supérieur, au-dessus de la mêlée, qui ne connaît pas les réalités ordinaires, qui fait partie du petit cercle d'initiés ("la France 
d'en haut", les élites...), est utile pour relier le lien de confiance entre les élus et les citoyens. Le jugement négatif verra surtout qu'on brade là l'image de la fonction politique, en essayant de la dissimuler derrière une vitrine populaire et démagogique, qu'on mise sur les individualités pour occulter les débats politiques de fond, au risque de renforcer des sentiments poujadistes et anti-politiques. On entre ici dans des appréciations qui excèdent les limites de l'analyse des programmes télévisés, et dans le registre des jugements subjectifs, ce qui n'est pas le rôle de cette recherche.

Les éléments du dossier sont présentés, tant pour les deux communautés belges que pour d'autres pays européens. Et ils permettent, entre autres, de montrer que la situation belge présente des programmes assez modérés en termes de spectacularisation. Cela est dû, tout d'abord, il faut le reconnaître, à des contraintes budgétaires qui ne permettent pas de réaliser des émissions à grand spectacle, quel qu'en soit le thème, comme peuvent le faire des chaînes de plus grands pays, dotées de budgets incomparables avec ceux de nos émetteurs publics et privés. C'est ensuite lié au fait que les responsables des programmes (directeurs de l'information, réalisateurs, journalistes) jouent de ces transgressions génériques avec prudence, avançant à pas comptés dans les aménagements de programmes, gardant en ligne de mire la frontière vie privée/vie publique, même si celle-ci se déplace peu à peu. De ce point de vue, même s'il y a concurrence entre les émetteurs, celle-ci n'est pas féroce au point de recourir à des surenchères dans l'offre de programmes. Il y a une volonté aussi affirmée de ne pas brusquer un public peut-être moins ouvert à ces transgressions (ou transformations) que dans d'autres lieux. Cela se marque par exemple dans la rareté de nos programmes satiriques, qui restent dans l'ensemble assez prudents, y compris avec le personnel politique.

Ce dossier trouve son origine dans une commande conjointe du CSA (Conseil Supérieur de l'Audiovisuel de la Communauté française de Belgique) et du Parlement de la Communauté française, organe législatif de tutelle des médias audiovisuels publics. Certains des membres de ces instances s'inquiétaient d'une forme de spectacularisation des émissions proposées durant la campagne électorale de 2004 et ont souhaité recevoir une analyse scientifique de ces émissions. Ils souhaitaient aussi que l'étude de la situation en Communauté française de Belgique soit comparée à celle d'autres régions et pays européens. 
Le rapport complet de la recherche est disponible sur le site web de ces deux institutions (http://www.csa.be et http://www.pcf.be). Le présent dossier s'intéresse pour une bonne part aux études des télévisions étrangères, en Flandre, en France, en Grande-Bretagne, en Italie et en Grèce. Cette confrontation est intéressante, en ce sens qu'elle révèle des positions contrastées, entre une Grèce qui découvre seulement les plaisirs de la personnalisation des débats politiques et une France qui semble revenir à des débats plus classiques, à côté d'une Italie où les questions de traitement médiatique sont largement tributaires des enjeux relatifs aux propriétaires des chaînes de télévision. Voici en tout cas une pièce à mettre au dossier d'une étude comparative de la question à un niveau européen, à laquelle nous souhaitons contribuer. 